\title{
The prevalence and the adding value of fetal MRI imaging in midline cerebral anomalies
}

\author{
Rehab M. E. Raafat' ${ }^{1}$ Tamer M. Abdelrahman ${ }^{2}$ and Mona A. F. Hafez ${ }^{3 *}$
}

\begin{abstract}
Background: Foetal MR imaging is widely accepted as an adjunct to foetal ultrasonography; however, there are many controversies regarding its importance and indications. Therefore, this study aimed to evaluate foetuses with different midline cerebral abnormalities, to determine the prevalence of these anomalies, to define the role of foetal $M R I$, and to compare MRI and ultrasound (US) result with postnatal MRI findings. Seventy-eight pregnant women who had foetuses with CNS abnormalities detected by sonogram were included. Foetuses with midline anomalies were selected and evaluated by anomaly scan foetal US, pre- and postnatal MRI.

Results: Midline brain anomalies were found in $47.4 \%$ of foetuses with brain anomalies. Holoprosencephaly was found in $24.3 \%$ of midline anomaly foetuses, corpus callosum abnormalities (ACC) were detected in $40.5 \%$, midline intracranial mass lesions in 2.7\%, and midline posterior fossa anomalies in 32.4\%. An agreement between MRI and US in the main diagnosis was in $56.76 \%$ of cases; MRI added information to US findings in $43.2 \%$ of cases, and US added information to MRI findings in $8.1 \%$ of cases.
\end{abstract}

Conclusion: In evaluating midline cerebral anomalies, US and MRI are complementary techniques. US is the primary survey, and MRI can add additional information and/or change the main diagnosis.

Keywords: Foetal ultrasound, Foetal MRI, Midline anomalies, Brain, Posterior fossa

\section{Background}

The prenatal diagnosis uses various non-invasive and invasive techniques to provide early information of any abnormality in a foetus [1]. Three-dimensional (3D) ultrasonography is the mainstay for foetal examination and detection of any abnormalities as early as possible [2]. The results of this examination are variable depending on the time of examination and the expertise of the examiner. The main difficulty in ultrasound (US) is in obtaining views that are not easily accessible such as the midsagittal plane of the foetal head that provide unique information on the corpus callosum and the cerebellar vermis $[3,4]$.

In recent studies, for the in utero evaluation of CNS pathological conditions, the use of foetal MR imaging has become widely accepted as an adjunct to foetal

\footnotetext{
* Correspondence: mona.fouad@kasralainy.edu.eg

${ }^{3}$ Diagnostic Radiology and Intervention Department, Cairo University,

Medical School, Kasr Al-Aini, Cairo, Egypt

Full list of author information is available at the end of the article
}

ultrasonography. Foetal MRI provides an accurate in utero diagnosis of most CNS anomalies [4,5]. Moreover, MRI allows evaluation of the foetal brain, particularly when a foetus is at increased risk for neurodevelopmental disabilities or documentation when an abnormality has been detected in the prenatal US [1].

Most midline brain anomalies obtained by the midsagittal plane of foetal MRI or US include commissural; corpus callosum; sellar, suprasellar, and pineal region; aqueductal stenosis; and posterior fossa, including the brainstem, cerebellum, and fourth ventricle, abnormalities [6].

In this study, we aimed to evaluate foetuses with different midline brain abnormalities, to determine the prevalence of these abnormalities, to define the role of foetal MRI, and to compare MRI results with US findings in these cases. 


\section{Methods}

Seventy-eight pregnant women were prospectively enrolled from the foetal medicine and prenatal diagnosis department of a University hospital in 1-year period. The ethical review board approved this study and written informed consent was obtained from each participant.

\section{Inclusion criteria}

Pregnant women who have foetuses with current CNS abnormalities by sonogram are referred for MRI assessment.

\section{Exclusion criteria}

Cases with other non-midline cerebral anomalies and cases with MRI contraindications as claustrophobia, cases with artificial prostheses or pacemakers contraindicated to MRI.

Cases with midline cerebral anomalies were selected. US images were evaluated by an experienced obstetrician and radiologist (25 and 10 years of experience, respectively), and MRI images were evaluated by two radiologists (12 and 10 years of experience). The results were then compared with postnatal MRI and follow-up of the cases.

Two-, three- and four-dimensional US assessments were performed for all cases, and the tomographic ultrasound imaging (TUI) technique was also used. The US machines used were Voluson 730 pro and E6 (GE Healthcare, Milwaukee, WI, USA) system) and Accuvix XQ (Medison, Korea) US machines equipped with 3.5$5 \mathrm{MHz} 3 \mathrm{D}$ transducers. The analysis of 3D volume datasets was performed either on the screen of the US machine or offline on a PC using dedicated 4D view software (GE Healthcare, Milwaukee WI, USA). Different display modalities were used according to the examiner preference and depending on the anomaly examined to facilitate the diagnosis process, for example, volume contrast imaging (VCI) or TUI in addition to the multi-planar view.

Foetal MRI was performed for all cases. The MRI machine used was a Philips MR machine Achieva XR 1.5 Tesla using sense Xl torso coil 16 elements and phased array; the machine was optimized for imaging of the abdomen and pelvis after a localizing gradient-echo sequence. Special sequences used included ultra-fast T2 weighted single-shot fast spin-echo balanced fast field echo (FFE), which is the manufacturer's name for the balanced steady-state free precession MRI pulse sequence (BSSFP); single-shot turbo spin-echo (TSE), and spoiled T1 gradient echo. MRI images were obtained according to foetal position in the axial, coronal and sagittal planes. An average of seven (5-12) sequences were obtained for every examination, scanning time/slice was less than $1 \mathrm{~s} /$ image, and the mean time was 7-15 min. Repetition of some sequences was required because some images were affected by foetal motion during acquisition or foetal motion between sequences resulted in images that were not in the true anatomic planes.

All live-born infants underwent postnatal evaluation by paediatric neurologists, and neonatal MRI was performed in 33 cases.

No significant time interval between prenatal US and MRI was observed for all cases performed on the same day.

\section{Results}

This prospective observational study included 78 pregnant women with CNS anomalies, and their ages ranged between 19 and 40 years old (mean $28.16 \pm 4.62$ SD years old). The gestational age at examination time was 1539 weeks (mean $27.90 \pm 6.19$ SD weeks). Thirteen cases (16.67\%) had positive consanguinity. Twenty-nine cases (37.2\%) were primigravida. Six cases $(7.7 \%)$ had a past history of foetal anomalies. None of the patients had a history of drug intake or radiation exposure.

After the evaluation of foetal MRI of all 78 cases, 41 cases were excluded due to the presence of other nonmidline CNS anomalies. Midline anomalies were found in $37(47.4 \%)$, their age ranged between 22 and 32 years old. The gestational age at examination time was 20-34 weeks. Of these cases, three $(8.1 \%)$ had positive consanguinity, and one case $(2.7 \%)$ had previously affected children. Six cases (16.2\%) were primigravida.

Holoprosencephaly was detected in 9/37 foetuses (24.3\%), including alobar holoprosencephaly in 7 cases (77.7\%), semilobar in 1 case (11.1\%), and lobar in 1 case (11.1\%). All alobar and semilobar holoprosencephaly cases were diagnosed by US and confirmed by MRI. The case of lobar holoprosencephaly was diagnosed by US as hydrocephalus, but MRI changed the diagnosis. US added the information of facial anomalies in 3 cases (Fig. 1).

Corpus callosum abnormalities (ACC) were detected in $15 / 37$ cases $(40.5 \%)$; complete corpus callosum agenesis was detected in 9/15 cases (60\%), and associated intracranial midline cysts were detected in $8 / 15$ foetuses (53.3\%) (Fig. 2). Partial ACC was found in 6 foetuses (40\%). Five cases of partial ACC were missed by US and diagnosed by MRI. Additionally, in a case of midline cyst, MRI changed the diagnosis to an arachnoid cyst and helped to assess the corpus callosum, which could not be seen by US.

A midline intracranial mass lesion was detected in $1 /$ 37 foetuses (2.7\%) (Fig. 3). US diagnosed an intracranial supratentorial heterogeneous mass lesion that was highly vascular in colour Doppler, with spreading hydrocephalic ventricles with a thin atrophic cerebral mantle. By 


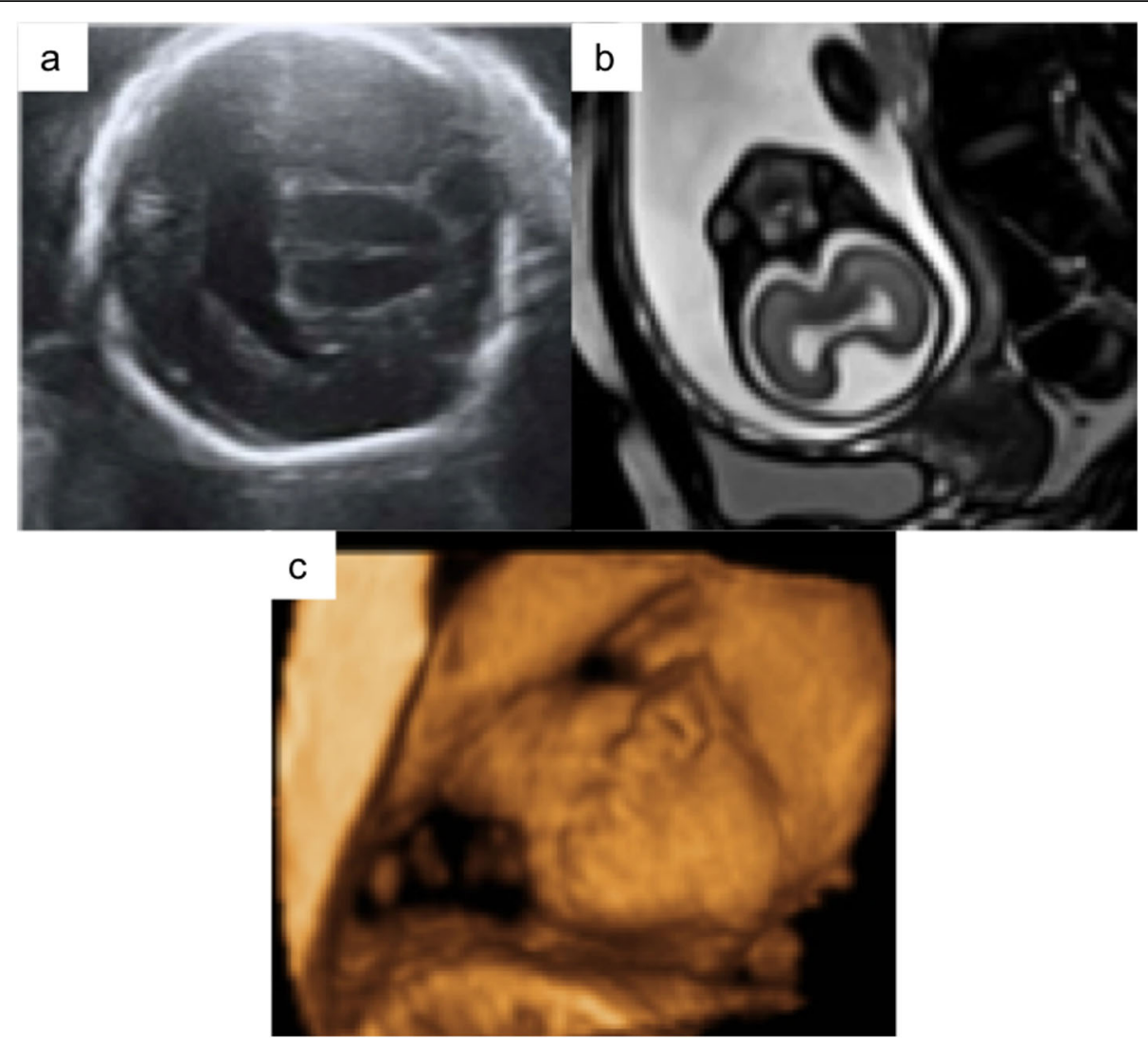

Fig. 1 A 20-year-old woman (gravida 1, para 0) at 18 weeks of gestation with an ultrasound (US) diagnosis of alobar holoprosencephaly. a 2D US demonstrated a small head, single ventricle, fused thalami, absent falx, and septum pellucidum; the findings were confirmed by MRI (b) at 20 weeks. 3D US (c) shows cyclops in the face, hypotelorism, and single nostril. Karyotype was offered but refused by the patient. The pregnancy was miscarried at 23 weeks

MRI, the mass was midline in location, showing multiple signal intensities. The mass was diagnosed and confirmed as craniopharyngioma by a histopathological postnatal operation.

Midline posterior fossa anomalies were noted in 12/37 cases (32.4\%). A total 9/12 cases (75\%) with cerebellar vermis hypoplasia were identified, and 3 cases were diagnosed as Dandy-Walker malformation. Two cases were diagnosed by US and confirmed by MRI, and 1 case was diagnosed by MRI. Dandy-Walker variant was identified in 4 cases, and all of them were diagnosed by MRI. One case $(8.3 \%)$ with Joubert syndrome was diagnosed by MRI, and 1 case (8.3\%) with Walker-Warburg syndrome was diagnosed by MRI (Fig. 4). One case (8.3\%) with aqueduct stenosis was diagnosed by MRI, and two cases (16.7\%) with mega cisterna magna were diagnosed by US and confirmed to be the solitary finding in MRI.

Other associated CNS anomalies were observed in 9/ 37 cases (24.3\%); eight cases had ventricular dilatation, six cases had midline shift, intraventricular haemorrhage in four cases, a cerebral cortical anomaly in one case, and spinal dysraphism in one case. However, extracerebral anomalies were observed in five cases (13.5\%).

The agreement between MRI and US in the main diagnosis was in 21/37 cases (56.76\%).

MRI changed the diagnosis and added information in $16 / 37$ cases (43.2\%); one case of rhombencephalosynapsis was identified by US, but it was normal by pre- and postnatal MRI. In other foetuses, ultrasonography revealed the molar tooth appearance of the brain stem, raising the possibility of Joubert syndrome, but DandyWalker variant was diagnosed in the MRI examination (pre- and postnatal). Thirteen cases with hydrocephalus in foetal US were ultimately diagnosed with partial ACC in 5 cases, lobar holoprosencephaly in 1 case, and posterior fossa anomalies in 7 cases.

3D US was superior to MRI in detecting midline facial anomalies (cyclops, hypertelorism, and single nostril), which were observed in three cases (8.1\%).

Four pregnancies were terminated or lost. In our study, in live births, pre- and postnatal MRI showed good agreement in 32/33 cases (97\%). 


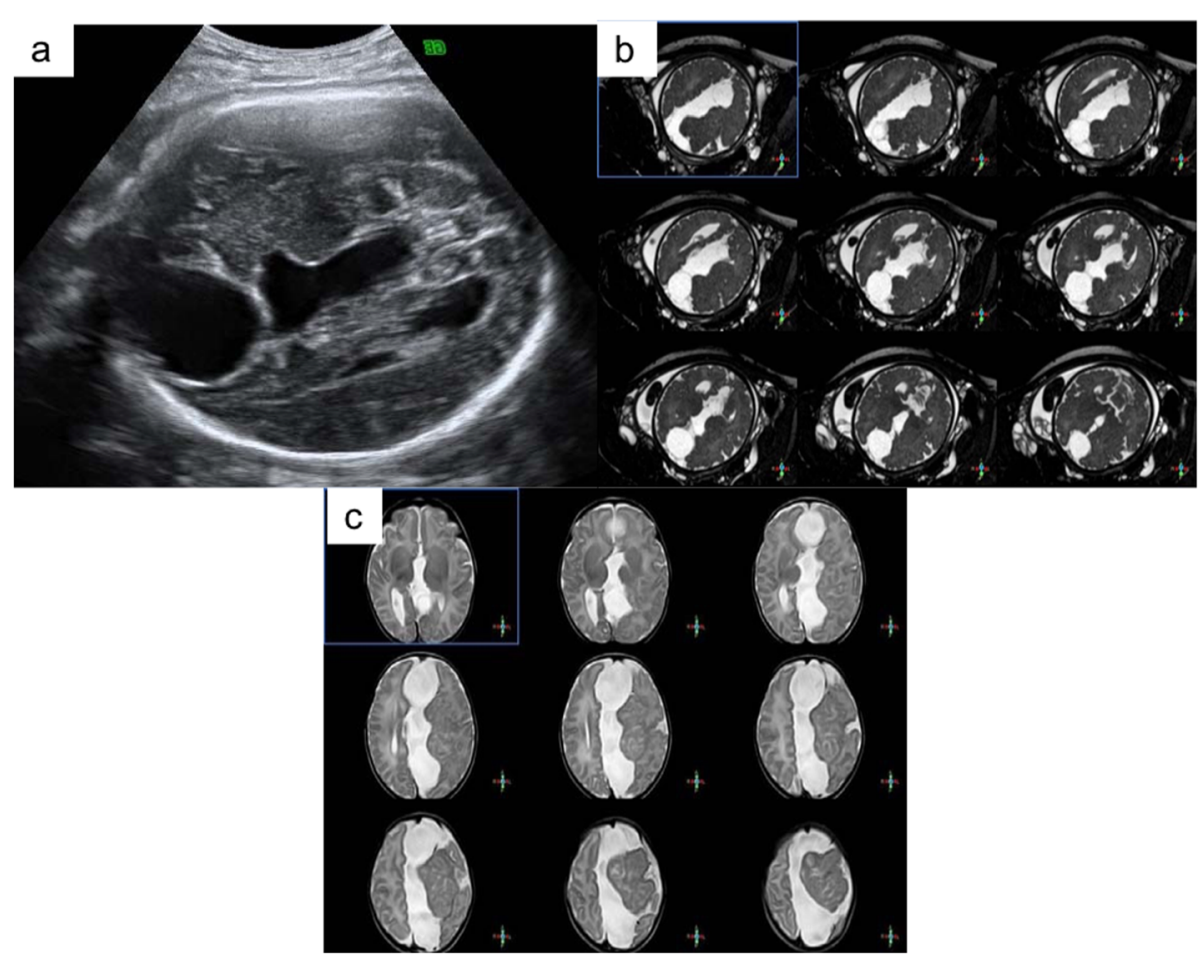

Fig. 2 A 27-year-old woman (gravida 3, para 2) with negative consanguinity and no history of a previously affected child was referred to the foetal unit. a Ultrasound (US) axial view shows midline interhemispheric cyst, evidence of callosal agenesis with the teardrop sign of the lateral ventricle. b Prenatal foetal BFFe WI axial cuts show the same lesions and superadded left-sided open and closed schizencephaly connected to an interhemispheric cyst. c Postnatal T2WI axial view confirms the prenatal MRI findings

The calculated prenatal MRI and US sensitivity, specificity and accuracy for holoprosencephaly, corpus callosum abnormalities, posterior fossa anomalies, and total midline cerebral anomaly cases are shown in Table 1.

\section{Discussion}

Suspicion of a CNS abnormality requires a multidisciplinary approach that includes imaging by prenatal US and MRI, as well as laboratory and genomics assessments. With the diagnosis of a congenital malformation, decisions are guided by the parent's personal background, education, and culture $[7,8]$.

Intrauterine MRI neuroimaging has multiple advantages, such as high soft-tissue contrast, a large field of view; additionally, it is unaffected by a suboptimal foetal position, oligohydramnios, ossified calvaria, and maternal habitus [9].

In this work, we evaluated the role and outcome of MRI and US as the primary imaging modalities in the evaluation of foetal midline cerebral anomalies in pregnant women. MRI provided additional information and changed the diagnosis to the previous US in 16 cases $43.2 \%$ of patients. However, US added information to MRI in 3 cases (8.1\%), and MRI agreed with US in 21 cases $(56.76 \%)$ and helped to terminate pregnancy in 4 cases $(10.8 \%)$. The diagnosis was confirmed by postnatal MRI in live birth.

There are great controversies regarding the prenatal US versus MRI, as Wagenvoort et al. [10] stated that foetal brain MRI added more information and is more accurate than foetal US. Additionally, Jarvis et al. [11] concluded that when foetal brain abnormalities are suspected on US, MR imaging can significantly contribute to the diagnostic pathway by clarifying findings and significantly increasing the detection rate of abnormalities, particularly in the midline and posterior fossa anomalies. However, dedicated neuro-sonography studies concluded that MRI is for the confirmation of diagnosis and that each modality provided additional/different information only in a minority of cases [12].

In our study, pre- and postnatal MRI showed good agreement in $97.3 \%$ of cases. Similarly, in Dhouib and colleagues' study [13], pre- and postnatal MRI data showed good agreement in $85 \%$ of cases, and there was disagreement with a prognostic impact in only $9 \%$ of cases.

The prenatal diagnosis of alobar holoprosencephaly is fairly easy; however, the recognition of the semilobar and lobar forms can present a diagnostic challenge. In some cases with US examination, the inability to detect the cavum septum pellucidum may be the only obvious 


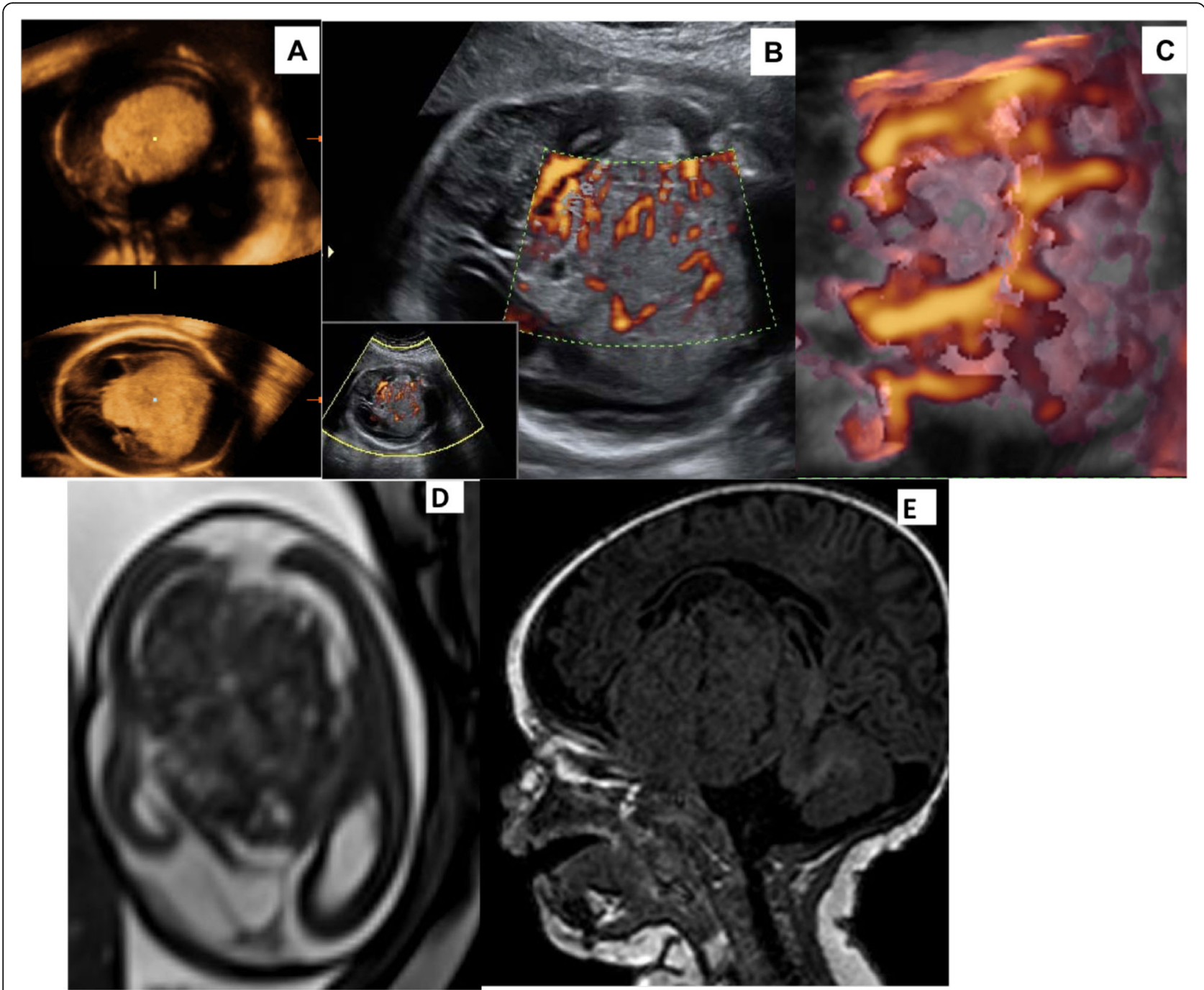

Fig. 3 A 23-year-old woman (primigravida), ultrasonography discovered an intracranial midline mass lesion during a prenatal anomaly scan at 20 weeks gestational age (GA). a Three-dimensional (3D) ultrasound (US) findings revealed midline heterogeneous mass lesions splaying across the ventricles. b, c Colour Doppler and 3D Angiography Doppler US images show that the lesion is highly vascular. d, e Prenatal MRI T2 BFFe WI and postnatal MRI sagittal T1WI show a supratentorial space-occupying mass lesion with heterogeneous signal intensity that was confirmed to be craniopharyngioma

finding at $18-20$ weeks. MRI is useful for further evaluation when US findings are unclear or to better define associated brain anomalies when the cavum septum pellucidum is absent [9].

In holoprosencephaly, 3D and 4D US surpass MRI in detecting associated midline facial anomalies. 3D and 4D US have been used as an adjunctive imaging modality to 2D ultrasonography to generate accurate detailed images of facial surface anatomy [14].

Prenatal detection of corpus callosal malformations present a challenge as the US signs may be very subtle or appear late after the second-trimester scans [15]. Direct visualization of the corpus callosum and its abnormalities by US is difficult; thus, we mainly depend on the indirect signs of callosal development (such as the absence of the cavum septum pellucidum, colpocephaly, and the inferior orientation of the medial hemispheric sulci). Foetal MRI is helpful in the evaluation of suspected corpus callosal anomalies by US because the corpus callosum can be visualized directly. Additionally, the indirect signs can be seen in foetal MRI. Many studies, such as Wagenvoort et al. [10] and Malinger et al. [16] concluded that MRI is superior to US, particularly in the diagnosis of callosal anomalies. In this study, five cases of partial callosal agenesis were missed by US and diagnosed by MRI.

The diagnostic accuracy for detecting failed commissuration in our study was $86.49 \%$ for US and $100 \%$ for MRI. Griffiths et al. [17] calculated accuracy of $34.2 \%$ for US and $94.9 \%$ for MRI with significant improvement in 


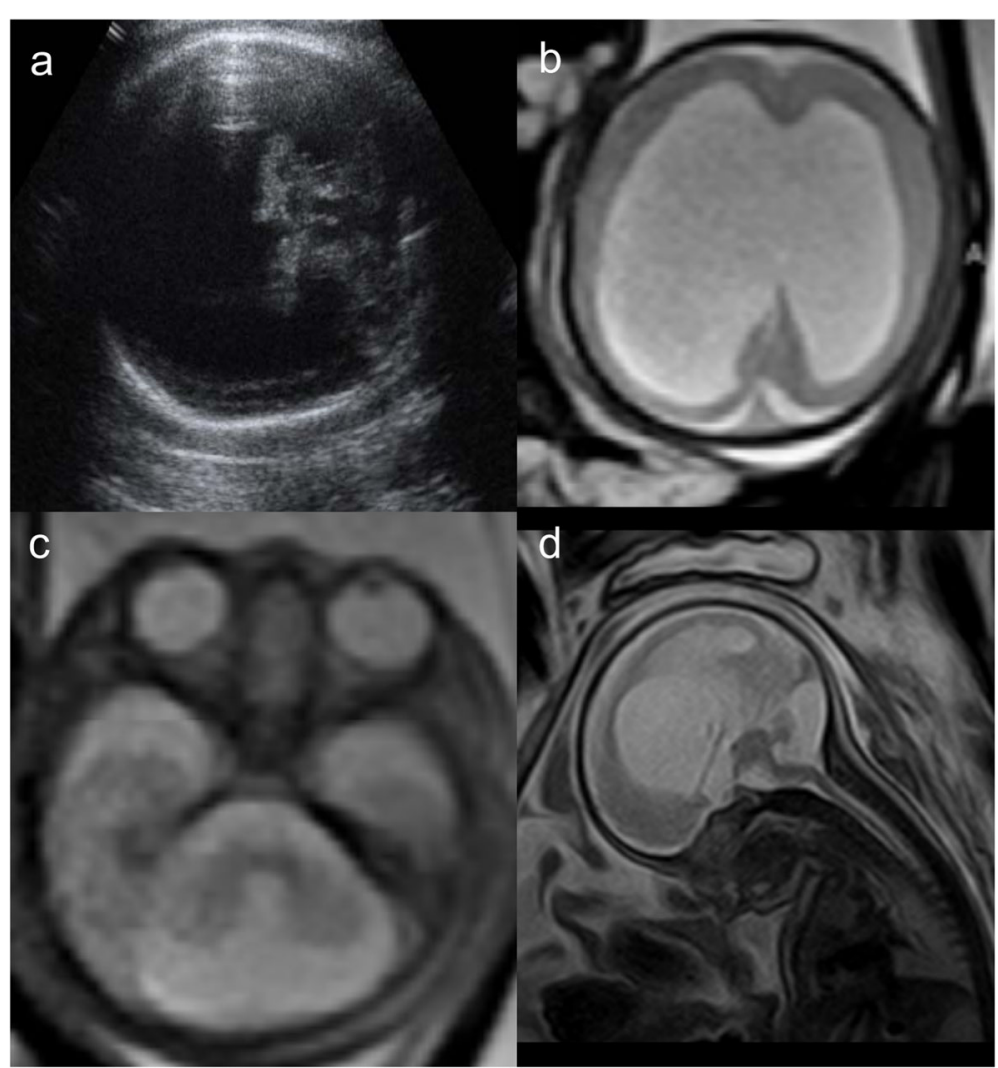

Fig. 4 A 32-year-old woman came to the feto-maternal unit at 34 weeks gestational age (GA) with hydrocephalus. Further ultrasound (US) neuroscan was limited due to the position of the foetus, and it showed hydrocephalus; additionally, the posterior fossa was small and inconspicuous (a). Foetal MRI was performed and showed hydrocephalic changes, cortical lissencephaly, (b) dilated retrocerebellar space associated with vermian and brain stem hypoplasia, with z-shape configuration of the brain stem (c, d). Confirmed to be Walker-Warburg syndrome

diagnostic confidence for MRI. Therefore, these authors suggest that any woman whose foetus has failed commissuration as the only intracranial finding detected on US should have an MRI examination for further evaluation.

MRI helps to define the nature of cerebral cysts [16]. In this study, we reported nine cases with midline interhemispheric cysts detected by US; MRI changed the diagnosis to an arachnoid cyst in one case, and in another case, we could not determine whether the large midline cyst was a cystic expansion of third ventricle or an associated interhemispheric cyst with the ACC by US.

Craniopharyngioma is a benign CNS tumour that may present with enlarged head circumference and hydrocephalus secondary to cerebrospinal fluid drainage obstruction. This tumour is mostly found in the suprasellar region. In US, craniopharyngioma appears as an intracranial large echogenic mass (differential with teratomas). MRI helps in the exact localization of the tumour and the assessment of the remaining brain structures [18]. In this study, there was a case with

Table 1 The calculated prenatal MRI and US sensitivity, specificity and accuracy for holoprosencephaly, corpus callosum abnormalities, posterior fossa anomalies, and total midline cerebral anomaly cases

\begin{tabular}{|c|c|c|c|c|c|c|}
\hline \multirow[t]{2}{*}{ Diagnosis } & \multicolumn{3}{|c|}{ Prenatal MRI } & \multicolumn{3}{|l|}{ US } \\
\hline & Sensitivity & Specificity & Accuracy & Sensitivity & Specificity & Accuracy \\
\hline Holoprosencephaly & $100 \%$ & $100 \%$ & $100 \%$ & $88.9 \%$ & $100 \%$ & $97.3 \%$ \\
\hline Corpus callosum abnormalities & $100 \%$ & $100 \%$ & $100 \%$ & $66.67 \%$ & $100 \%$ & $86.49 \%$ \\
\hline Posterior fossa anomalies & $91.67 \%$ & $100 \%$ & $97.30 \%$ & $41.67 \%$ & $92.59 \%$ & $76.92 \%$ \\
\hline Total & $97.3 \%$ & $100 \%$ & $98.72 \%$ & $56.76 \%$ & $95.35 \%$ & $77.50 \%$ \\
\hline
\end{tabular}


craniopharyngioma, and US was superior in the detection of intra-tumoural vascularity by colour Doppler imaging; however, MRI helped in the assessment of the posterior fossa and the remaining brain structures (Fig. 3).

Posterior fossa anomalies comprise various malformations, such as Dandy-Walker malformation, various causes of vermian hypoplasia or aplasia, persistent Blake's pouch, mega cisterna magna, and cerebellar hypoplasia [8]. The overall diagnostic accuracy for detecting posterior fossa abnormalities in our study was $97.3 \%$ for prenatal MRI and 76.92\% for US. Griffiths et al. [19] showed comparable results with $65.4 \%$ diagnostic accuracy for US and $87.7 \%$ for MRI. Additionally, any woman whose foetus has a posterior fossa abnormality on US should have MRI for further evaluation to improve diagnostic accuracy and confidence.

US and foetal MRI are complementary techniques; when using one of these imaging modalities, we should be aware of their main advantages and limitations. We should determine the imaging strategy on a case-by-case basis depending on the maternal body habitus, the foetal presentation, and the type of abnormalities suspected. Notably, accurate prenatal diagnosis is of the utmost importance for prenatal counselling [20].

The results of the MERIDIAN cohort studies [21] strongly support the routine clinical use of intrauterine MRI as an adjunct to US when assessing foetuses with structural brain abnormalities. Therefore, intrauterine MRI was potentially beneficial when included in the diagnostic pathway for prenatal structural midline brain anomalies. This technique improves diagnostic accuracy and confidence for foetal brain anomalies and leads to better counselling and management changes in a high proportion of cases [3].

The main limitation of this study is the small number of the study group, as it would be more beneficial if it was performed in a large scale multicentre study.

\section{Conclusion}

In the evaluation of midline cerebral anomalies, US and MRI are complementary techniques. US is the main primary survey and is especially beneficial for facial anomalies, and MRI can add additional information, especially for partial corpus callosal abnormalities and posterior fossa anomalies.

\section{Abbreviations}

3, 4D: Three, four-dimensional; ACC: Agenesis of the corpus callosum; CNS: Central nervous system; MRI: Magnetic resonance imaging; TUI: Tomographic ultrasound imaging; US: Ultrasound; VCI: Volume contrast imaging

\section{Acknowledgements}

We thank obstetrics and gynaecology team for their case referral, neurogenetics team for cases follow-up, participants who trusted us for their patience and support and MRI technicians for their assistance.

\section{Authors' contributions}

RR suggests and develops the research idea, reviewing literature, data collection and analysis, performing the examinations, organizing the results, and share in write and revise the manuscript. TA contributed to data collection and analysis. MH contributed to data analysis, reviewing literature, perform statistical analysis, organizing results, write and revise the manuscript, manuscript editing. All authors read and approved the final manuscript.

Funding

This study had no funding from any resource.

\section{Availability of data and materials}

The datasets used and analysed during the current study are available from the corresponding author on reasonable request.

\section{Ethics approval and consent to participate}

This study was approved by the Research Ethics Committee of the Faculty of Medicine at Cairo University in Egypt with ethical committee reference number I 071015. All patients included in this study gave written informed consent to participate in this research.

\section{Consent for publication}

All patients included in this research gave written informed consent to publish the data contained within this study.

\section{Competing interests}

The authors declare that they have no competing interests.

\section{Author details}

${ }^{1}$ Diagnostic Radiology and Intervention Department, Sheikh zayed Al Nahyan Hospital, Cairo, Egypt. Diagnostic Radiology and Intervention Department, National Cancer Institute, Cairo University, Cairo, Egypt. ${ }^{3}$ Diagnostic Radiology and Intervention Department, Cairo University, Medical School, Kasr Al-Aini, Cairo, Egypt.

Received: 12 July 2019 Accepted: 21 January 2020

Published online: 10 February 2020

\section{References}

1. Glenn OA (2010) MR imaging of the fetal brain. Pediatr Radiol 40(1):68-81

2. Lee W, Chang CH, Cheng YC, Ko HC, Chang FM (2012) A review of threedimensional ultrasound applications in fetal growth restriction. J Med Ultrasound 20(3):142-149

3. Pilu G, Segata M, Ghi T et al (2006) Diagnosis of midline anomalies of the fetal brain with the three-dimensional median view. Ultrasound Obstet Gynecol 27:522-529

4. Griffiths PD, Bradburn M, Campbell MJ et al (2017) Use of MRI in the diagnosis of fetal brain abnormalities in utero (MERIDIAN): a multicentre, prospective cohort study. Lancet 389:538-546

5. Peruzzi P, Corbitt RJ, Raffel C (2010) Magnetic resonance imaging versus ultrasonography for the in utero evaluation of central nervous system anomalies. Neurosurg Pediatr 6(4):340-350

6. Choudhri AF, Cohen HL, Siddiqui A et al (2018) Twenty-five diagnoses on midline images of the brain: from fetus to child to adult. RadioGraphics 38 : 218-235

7. De Keersmaecker B, Claus F, De Catte $L$ (2011) Imaging the fetal central nervous system. Facts Views Vis ObGyn 3(3):135-149

8. Snoek R, Albers MEWA, Mulder EJH et al (2018) Accuracy of diagnosis and counseling of fetal brain anomalies prior to 24 weeks of gestational age. J Matern Fetal Neonatal Med 31(16):2188-2194

9. Winter TC, Kennedy AM, Woodward PJ (2015) Holoprosencephaly: a survey of the entity, with embryology and fetal imaging. RadioGraphics 35:275-290

10. Wagenvoort AM, Bekker MN, Go ATII, Vandenbussche FB, van Buchem VJ, van Vugt JM (2000) Ultrafast scan magnetic resonance in prenatal diagnosis. Fetal Diagn Ther 15:364-372

11. Jarvis D, Mooney C, Cohen J, Papaioannou D, Bradburn M, Sutton A, Griffiths PD (2017) A systematic review and meta-analysis to determine the contribution of MR imaging to the diagnosis of foetal brain abnormalities in utero. Eur Radiol 27(6):2367-2380 
12. Pooh RK, Pooh KH (2011) Fetal neuroimaging by transvaginal 3D ultrasound and MRI. Donald School J Ultrasound Obst Gynecol 5(1):23-32

13. Dhouib A, Blondiaux E, Moutard ML et al (2011) Correlation between preand postnatal cerebral magnetic resonance imaging. Ultrasound Obstet Gynecol 38:170-178

14. Saleem SN (2014) Fetal MRl: an approach to practice: a review. J Adv Res 5(5):507-523

15. Lipa M, Pooh RK, Wielgoś M (2017) Three-dimensional neurosonographya novel field in fetal medicine. Ginekol Pol 88(4):215-221

16. Malinger G, Ben-Sira L, Lev D et al (2014) Fetal brain imaging: a comparison between magnetic resonance imaging and dedicated neurosonography. Ultrasound Obstet Gynecol 23:333-340

17. Griffiths PD, Brackley K, Bradburn M et al (2017) Anatomical subgroup analysis of the MERIDIAN cohort: failed commissuration. Ultrasound Obstet Gynecol 50:753-760

18. Milani HJ, Júnior EA, Cavalheiro $S$ et al (2015) Fetal brain tumors: prenatal diagnosis by ultrasound and magnetic resonance imaging. World J Radiol 7(1):17-21

19. Griffiths PD, Brackley K, Bradburn M et al (2017) Anatomical subgroup analysis of the MERIDIAN cohort: posterior fossa abnormalities. Ultrasound Obstet Gynecol 50:745-752

20. Blondiaux E, Garel C (2013) Fetal cerebral imaging - ultrasound vs. MRI: an update. Acta Radiol 54(9):1046-1054

21. Griffiths PD, Bradburn M, Campbell MJ et al (2017) Change in diagnostic confidence brought about by using in utero MRI for fetal structural brain pathology: analysis of the MERIDIAN cohort. Clin Radiol 72:451-457

\section{Publisher's Note}

Springer Nature remains neutral with regard to jurisdictional claims in published maps and institutional affiliations.

\section{Submit your manuscript to a SpringerOpen ${ }^{\circ}$ journal and benefit from:}

- Convenient online submission

- Rigorous peer review

- Open access: articles freely available online

- High visibility within the field

- Retaining the copyright to your article

Submit your next manuscript at $\boldsymbol{\nabla}$ springeropen.com 